\title{
Impacto de la Pandemia por SARS-CoV2 sobre la Educación
}

\author{
Impact of the SARS-CoV2 Pandemic on Education \\ Impacto da pandemia de SARS-CoV2 na educação
}

\author{
Carlos Jiménez-Sánchez \\ Universidad Nacional \\ ROR:: https://ror.org/01t466c14 \\ Escuela de Medicina Veterinaria \\ Laboratorio de Virología \\ Heredia, Costa Rica \\ carlos.jimenez.sanchez@una.ac.cr \\ https://orcid.org/0000-0002-9544-5285
}

\begin{abstract}
Resumen: Se describe el impacto de la pandemia por SARS-CoV2 sobre la educación y se propone algunos escenarios donde la Universidad Nacional puede contribuir a mitigar el daño y a participar en la construcción del escenario postpandémico.
\end{abstract}

Palabras claves: Pandemia; educación; mitigación; SARS-CoV2.

\begin{abstract}
It describes the impact of the SARS-CoV2 pandemic on education and proposes some scenarios where the Universidad Nacional can contribute to mitigate the damage and participate in the construction of the post-pandemic scenario.
\end{abstract}

Keywords: Pandemic; education; mitigation; SARS-CoV2.

Resumo: Descreve brevemente o impacto da pandemia da SRA-CoV2 na educação e propõe alguns cenários em que a Universidade Nacional pode contribuir para mitigar os danos e participar na construção do cenário pós-pandémico.

Palavras-chaves: Pandemi; educação; mitigação; SARS-CoV2.

Recibido: 15/07/2020 Aceptado: 04/08/2020

\section{Introducción}

Los efectos de la pandemia, provocada por el SARS-CoV2, sobre la salud pública, la economía y la educación son extensos. Este texto pretende analizar, someramente, este último impacto y proponer algunos escenarios donde la Universidad Nacional, Costa Rica, contribuya a mitigar el daño y participe proactivamente en la construcción del escenario postpandémico. 
https://doi.org/10.15359/ree.24-S.1

https://www.revistas.una.ac.cr/index.php/educare

educare@una.ac.cr

\section{Desarrollo}

El 11 de marzo de 2020, la Organización Mundial de la Salud (OMS) declaró la COVID-19, causada por el SARS-CoV2, como pandemia (OMS, 2020). Al día de hoy, 15 de julio de 2020, la COVID-19 se ha extendido a 213 países y territorios, ha afectado a 13,662,824 personas y causado el fallecimiento de 585,619 seres humanos (Worldometer, 2020). En un afán por controlar la diseminación de la enfermedad, muchos países implementaron, el distanciamiento social, el cual conlleva al enclaustramiento, al cierre de empresas públicas y privadas así como de escuelas, colegios y universidades. En este último grupo, la UNESCO (2020) ha estimado que, a nivel mundial, al menos 1,184,126,508 de estudiantes se han visto afectados directamente en su proceso de aprendizaje, pero además, se ven perjudicados sus familiares, docentes y administrativos, y conlleva a efectos negativos en la economía de las naciones, siendo mas fuerte el impacto en los pobres y necesitados. En Costa Rica al menos 1,300,000 de estudiantes de enseñanza primaria, secundaria y universitaria dejaron de recibir clases presenciales. Con el propósito de mantener activos los ciclos lectivos, muchas instituciones educativas migraron su proceso de enseñanza-aprendizaje hacia la virtualidad. Sin embargo, la pandemia ha desnudado las enormes diferencias que subyacían en la poca disponibilidad de equipo adecuado y en la cobertura de internet, una brecha digital muy heterogénea y difícil de subsanar.

Como parte de las instituciones estatales de educación superior, la Universidad Nacional (UNA), con su misión de formar profesionales humanistas, tiene la oportunidad de apoyar el esfuerzo nacional para superar la pandemia y de forjar un futuro postpandémico mejor, echando mano de los recursos científicos y tecnológicos que tiene a su disposición. En primer lugar la institución puede actuar llevando educación basada en evidencia científica a la población, que permita a los ciudadanos entender de forma precisa, los riesgos, las consecuencias de sus actos y las posibilidades que tienen para evitar enfermarse. En segundo lugar la UNA cuenta con una amplia experiencia en la mediación pedagógica, posee una numerosa oferta académica a nivel de grado y posgrado y un enorme capital humano en muchas disciplinas (economía, política, psicología, veterinaria, agronomía, matemática, epidemiología, química, toxicología, vulcanología, sismología, biología, música, danza, teatro, literatura, administración, filosofía, ciencias ecuménicas, informática, turismo sostenible, hidrología, ciencias del movimiento humano y calidad de vida, educación, secretariado, desarrollo sostenible, topografía, geodesia, gestión de riesgos, recursos naturales, territorio y ambiente, manejo de recursos marinos y dulce-acuícolas, etc.), todo este acervo puede verterse a la sociedad costarricense desde las unidades académicas, facultades, sedes e institutos o a través de colaboración con los ministerios gubernamentales. En este rubro, acorde con la misión y visión institucionales, debe participar, también, la población estudiantil de forma tal, que la situación excepcional que genera la pandemia, se convierta en una situación excepcional de aprendizaje. 


\section{Conclusión}

Los efectos de la pandemia por SARS-CoV2 ofrecen una oportunidad para ejecutar esfuerzos conjuntos basados en la racionalidad, la solidaridad y el humanismo, que contribuyan a mitigar las consecuencias de la COVID-19 y a preparar un mejor escenario pospandémico.

Nota general: Corrección de estilo y traducciones a cargo del autor.

\section{Referencias}

Organización Mundial de la Salud. (2020). COVID-19: cronología de la actuación de la OMS. Autor. https://www.who.int/es/news-room/detail/27-04-2020-who-timeline---covid-19

UNESCO. (2020). Education: From disruption to recovery. Autor. https://en.unesco.org/covid19/ educationresponse/

Worldometer. (2020). COVID-19 coronavirus pandemic. Autor. HTTPS://WWW.WORLDOMETERS. INFO/CORONAVIRUS/?FBCLID=IWAR1OAZCSYWCWF7ZUEBMJCYLSD0Q6B VO6ANXWZFDQG09D50-YC609LJEX6K 